\title{
THE CAPELLI IDENTITY FOR GRASSMANN MANIFOLDS
}

\author{
SIDDHARTHA SAHI
}

\begin{abstract}
The column space of a real $n \times k$ matrix $x$ of rank $k$ is a $k$-plane. Thus we get a map from the space $X$ of such matrices to the Grassmannian $\mathbb{G}$ of $k$-planes in $\mathbb{R}^{n}$, and hence a $G L_{n}$-equivariant isomorphism

$$
C^{\infty}(\mathbb{G}) \approx C^{\infty}(X)^{G L_{k}} .
$$

We consider the $O_{n} \times G L_{k}$-invariant differential operator $C$ on $X$ given by

$$
C=\operatorname{det}\left(x^{t} x\right) \operatorname{det}\left(\partial^{t} \partial\right), \quad \text { where } x=\left(x_{i j}\right), \partial=\left(\frac{\partial}{\partial x_{i j}}\right) .
$$

By the above isomorphism, $C$ defines an $O_{n}$-invariant operator on $\mathbb{G}$.

Since $\mathbb{G}$ is a symmetric space for $O_{n}$, the irreducible $O_{n}$-submodules of $C^{\infty}(\mathbb{G})$ have multiplicity 1 ; thus, $O_{n}$-invariant operators act by scalars on these submodules. Our main result determines these scalars for a general class of such operators including $C$. This answers a question raised by Howe and Lee and also gives new Capelli-type identities for the orthogonal Lie algebra.
\end{abstract}

\section{INTRODUCTION}

1.1. The classical Capelli identity. Let $\left\{z_{i j}: 1 \leq i, j \leq n\right\}$ be $n^{2}$ variables and let $\partial_{i j}=\frac{\partial}{\partial z_{i j}}$ be the corresponding partial derivatives. Consider the $n \times n$ matrices $z=\left(z_{i j}\right)$ and $\partial=\left(\partial_{i j}\right)$. The classical Capelli identity [3] is the following identity of differential operators

$$
\operatorname{det}(z) \operatorname{det}(\partial)=\operatorname{det}\left(z^{t} \partial+(n-i) \delta_{i j}\right) .
$$

Here $\delta_{i j}$ is the Kronecker $\delta$ function, and the (noncommuting) determinant on the right is defined as follows:

$$
\operatorname{det}\left(a_{i j}\right)=\sum_{w \in S_{n}} \operatorname{sgn}(w) a_{w(1), 1} \cdots a_{w(n), n} .
$$

This identity plays a key role in the work of Herman Weyl 20, and others on classical invariant theory. It has also appeared more recently in the work of Atiyah, Bott, and Patodi [1] on the index theorem. We refer the reader to [7] and [2] for a discussion of the background of this identity. In the early $90 \mathrm{~s}$, the identity was generalized considerably by B. Kostant and the author ([13, [14, [19]) and by Howe and Umeda [10] to the context of Jordan algebras and multiplicity free actions. Since then there has been considerable interest in generalizations of Capelli-type identities, we refer the reader to [4, [11, 12, [15, [16, [17, 18] and the references therein.

The representation-theoretic meaning of (1.1) was explained in [7. The polynomial algebra $\mathbb{C}\left[z_{i j}\right]$ is the affine coordinate ring of the space $M_{n, n}$ of $n \times n$ matrices.

Received by the editors April 28, 2012 and, in revised form, December 13, 2012.

2010 Mathematics Subject Classification. Primary 22E46, 43A90.

Key words and phrases. Capelli identity, Grassmannian, invariant differential operator. 
The group $G L_{n} \times G L_{n}$ acts on $M_{n, n}$ by left and right multiplication, and hence we get a representation $\pi$ on $\mathbb{C}\left[z_{i j}\right]$. The operator $\operatorname{det}(z) \operatorname{det}(\partial)$ on the left of (1.1) is a $G L_{n} \times G L_{n}$ invariant differential operator on $\mathbb{C}\left[z_{i j}\right]$. It follows from $\left(G L_{n}, G L_{n}\right)$ duality 7 that such an operator is necessarily of the form $\pi(\Omega)$ for some element $\Omega$ in the center of the enveloping algebra of $\mathfrak{g l}_{n} \oplus \mathfrak{g l}_{n}$. Note, however, that the element $\Omega$ is not unique, and the expression on the right of (1.1) merely identifies one possible choice of $\Omega$. Indeed, the entries of the matrix $\left(z^{t} \partial\right)_{i j}=\sum_{p} z_{p i} \partial_{p j}$ come from the right action of $G L_{n}$ alone, and one can find other similar expressions in terms of the matrix entries of the left action $\left(z \partial^{t}\right)_{i j}=\sum_{p} z_{i p} \partial_{j p}$, or the diagonal action $\left(z \partial^{t}-z^{t} \partial\right)_{i j}$, etc.

There is, however, an associated eigenvalue problem that does admit a unique answer. Again, by $\left(G L_{n}, G L_{n}\right)$ duality, or in this case by the Peter-Weyl theorem, one knows that $\mathcal{P}_{n, n}$ decomposes as a direct sum of $G L_{n} \times G L_{n}$-modules $\mathcal{P}_{n, n}=$ $\bigoplus_{\lambda}\left(V^{\lambda} \otimes V^{\lambda}\right)$ where $\lambda$ ranges over partitions of length $\leq n$ and $V^{\lambda}$ is the $G L_{n}$ representation with highest weight $\lambda$. By Schur's lemma $\operatorname{det}(z) \operatorname{det}(\partial)$ acts by a scalar $p(\lambda)$ on $V^{\lambda} \otimes V^{\lambda}$ and one may ask for an explicit formula for this scalar. This turns out to have a rather pretty answer:

$$
p(\lambda)=\prod_{i=1}^{n}\left(\lambda_{i}+n-i\right) .
$$

As explained in [13, the eigenvalue formula (1.2) implies (1.1) via the HarishChandra homomorphism. Moreover, this point of view leads to a much larger class of identities in the context of Jordan algebras ([13, [14, [19]).

1.2. The Capelli identity for Grassmann manifolds. In 9] Howe and Lee pose a similar eigenvalue problem in the context of Grassmannians. In order to describe this problem we need some notation. Fix integers $k, l$ with $k<l$ and write $n=k+l$. Let $\mathbb{G}=\mathbb{G}_{n, k}$ denote the Grassmannian of $k$ planes in $\mathbb{C}^{n}$, and let $\mathcal{R}=\bigoplus_{m=0}^{\infty} \mathcal{R}_{m}$ be its homogeneous coordinate ring. The ring $\mathcal{R}$ admits an explicit description via the Plücker imbedding that we now recall briefly, referring the reader to [5, Ch. 9] for details. Let $\mathcal{P}=\mathbb{C}\left[z_{11}, \ldots, z_{n k}\right]$ be the coordinate ring of the space $M_{n, k}$ of $n \times k$ matrices, regarded as a $G L_{n} \times G L_{k}$-module. Then $\mathcal{R}$ is the subalgebra of $\mathcal{P}$ generated by the determinants of $k \times k$ minors. Alternatively we have

$$
\mathcal{R}=\mathcal{P}^{S L_{k}}, \mathcal{R}_{m}=\mathcal{P}^{\left(G L_{k}, \operatorname{det}^{m}\right)}
$$

with the usual notation for invariants and equivariants for the right action of $G L_{k}$ on $M_{n, k}$. This description also shows that each $\mathcal{R}_{m}$ is naturally a module under the left action of $G L_{n}$. By (e.g. [7]) $\mathcal{R}_{m}$ has a multiplicity-free $O_{n}$-decomposition,

$$
\mathcal{R}_{m}=\bigoplus_{\mu} \mathcal{R}_{m}^{\mu}
$$

and the occurring summands are indexed by partitions of length $\leq k$, whose parts are less than $m$ and have the same parity as $m$. In other words, $\mu$ ranges over integer sequences $\mu=\left(\mu_{1}, \cdots, \mu_{k}\right)$ satisfying

$$
m \geq \mu_{1} \geq \cdots \geq \mu_{k} \geq 0, \mu_{i} \equiv m(\bmod 2) \text { for all } i .
$$

Consider now the $n \times k$ matrices $z=\left(z_{i j}\right)$ and $\partial=\left(\frac{\partial}{\partial z_{i j}}\right)$ and define

$$
\gamma(z)=\operatorname{det}\left(z^{t} z\right), L=\operatorname{det}\left(\partial^{t} \partial\right) .
$$


The differential operator $L$ and the multiplication operator $\gamma$ are $O_{n}$-invariant and transform under $G L_{k}$ by the characters $\operatorname{det}^{-2}$ and $\operatorname{det}^{2}$, respectively. Thus they define maps

$$
\gamma: \mathcal{R}_{m}^{\mu} \rightarrow \mathcal{R}_{m+2}^{\mu}, L: \mathcal{R}_{m}^{\mu} \rightarrow \mathcal{R}_{m-2}^{\mu} .
$$

It follows that for any two integers $d, d^{\prime} \geq 0$, the operator

$$
C_{d, d^{\prime}}=\gamma^{d} \circ L^{d+d^{\prime}} \circ \gamma^{d^{\prime}}
$$

is an $O_{n}$-invariant operator on $\mathcal{R}_{m}^{\mu}$. By Schur's lemma $C_{d, d^{\prime}}$ acts by a scalar on $\mathcal{R}_{m}^{\mu}$, and Howe and Lee [9] ask for an explicit formula for these scalar eigenvalues. To be precise they consider only the operator $L \gamma=C_{0,1}$, but the general case is not much more difficult as we explain below. For $k=1$ the problem reduces to the classical theory of harmonic polynomials, and Howe and Lee [9] solve the problem for $k=2$ by an explicit calculation. Their calculation is elementary but fairly intricate and it is unclear how to extend it for $k \geq 3$.

In this paper we solve the eigenvalue problem for all $k$ using completely different methods. Let us define the following $k$-tuple of rational numbers

$$
\rho=\left(\rho_{1}, \ldots, \rho_{k}\right) \in \mathbb{Q}^{k}, \rho_{i}=\frac{n}{2}-i .
$$

Also, let $s$ and $\tau=\left(\tau_{1}, \ldots, \tau_{k}\right)$ be $k+1$ indeterminates and define polynomials

$$
q_{1,0}(s, \tau)=\prod_{i=1}^{k}\left(s^{2}-\tau_{i}^{2}\right), q_{d, d^{\prime}}(s, \tau)=\prod_{j=0}^{d+d^{\prime}-1} q_{1,0}\left(s+2 d^{\prime}-2 j, \tau\right) .
$$

Our main result is as follows:

Theorem 1.1. $C_{d, d^{\prime}}$ acts on $\mathcal{R}_{m}^{\mu}$ by the scalar $q_{d, d^{\prime}}\left(m+\rho_{1}, \mu+\rho\right)$.

Specializing to $k=2, d=0, d^{\prime}=1$, we see that $q_{0,1}\left(m+\rho_{1}, \mu+\rho\right)$ equals

$$
\left(m-\mu_{1}+2\right)\left(m+\mu_{1}+n\right)\left(m-\mu_{2}+3\right)\left(m+\mu_{2}+n-1\right),
$$

which agrees with Theorem 6.1 of [9, using formulas in [9, p. 356] for $\lambda=\mu$.

We now briefly sketch the proof of Theorem 1.1. In the next section, we reduce the problem to the special case of the operator $C=C_{1,0}=\gamma L$, and prove a simple lemma about the kernel of $C$. We also explain how to imbed the problem into the more general real analytic setting of the symmetric space $Y=S O_{n}(\mathbb{R}) /\left[S O_{k}(\mathbb{R}) \times S O_{l}(\mathbb{R})\right]$. The operator $C$ gives rise to a family of invariant operators $C_{s}$ on $Y$. The structure of invariant differential operators on a symmetric space is given by the Harish-Chandra homomorphism. In Sections 3 and 4 we recall this theory and specialize it to $Y$. In Section 5 we prove the eigenvalues of $C_{s}$ are given by a polynomial function with suitable symmetry properties. Finally, in Section 6 we use this polynomiality result, together with the knowledge of the kernel of $C$, to prove Theorem 2.1 up to an overall constant, which we then show to be 1 by an auxiliary computation.

In the appendix, we combine the results of this paper with those of 11 and [15] to obtain identities of invariant differential operators on the symmetric space $S O(p+q) / S O(p) \times S O(q)$. The main theorem also leads to a new inversion formula for the Radon transform on Grassmannians, which we plan to discuss in a subsequent paper. 


\section{REDUCtion of the PROBlem}

We first explain how deduce Theorem 1.1 from a special case. Define

$$
C=C_{1,0}=\gamma L \text {. }
$$

Theorem 2.1. $C$ acts on $\mathcal{R}_{m}^{\mu}$ by the scalar $q_{1,0}\left(m+\rho_{1}, \mu+\rho\right)$.

Proof of Theorem 1.1. We abbreviate $q(m)=q_{1,0}\left(m+\rho_{1}, \mu+\rho\right)$. If $v$ in $\mathcal{R}_{m}^{\mu}$, then $\gamma^{d^{\prime}} v \in \mathcal{R}_{m+2 d^{\prime}}^{\mu}$, and by Theorem 2.1 we get

$$
L\left(\gamma^{d^{\prime}} v\right)=q\left(m+2 d^{\prime}\right) \gamma^{d^{\prime}-1} v
$$

Iterating this we get

$$
L^{d+d^{\prime}} \gamma^{d^{\prime}} v=\left[\prod_{i=0}^{d+d^{\prime}-1} q\left(m+2 d^{\prime}-2 i\right)\right] \gamma^{-d} v
$$

We multiply both sides by $\gamma^{d}$ to get the result.

We now sketch the proof of Theorem 2.1. The following simple result on the kernel of $C$ plays a key role.

Lemma 2.2. If $\mu_{1}=m$, then $C$ acts by 0 on $\mathcal{R}_{m}^{\mu}$.

Proof. As noted above, $L: \mathcal{R}_{m}^{\mu} \rightarrow \mathcal{R}_{m-2}^{\mu}$. If $\mu_{1}=m$, then $\mathcal{R}_{m-2}^{\mu}=0$, thus $L$ acts by 0 on $\mathcal{R}_{m}^{\mu}$ and so does $C=\gamma L$.

In the next few sections we establish some general results about the eigenvalues of operators such as $C$, which enable us to deduce Theorem 2.1 from the above lemma. The key result is Proposition 5.3 below, which says that these eigenvalues are given by a polynomial function in the parameters $m, \mu$ with certain symmetry properties. In order to prove this proposition, it is important to extend the eigenvalue problem to a larger real analytic setting that we now explain.

The (holomorphic) polynomials in $\mathcal{P}$ and $\mathcal{R}$ are determined by their restrictions to the space $M_{n, k}(\mathbb{R})$ of real $n \times k$ matrices, and even to the open subset

$$
X=M_{n, k}^{\prime}(\mathbb{R})
$$

of rank $k$ matrices. We will write $x_{i j}$ for the (real) coordinate functions on $M_{n, k}(\mathbb{R})$ and $X$, and regard $\mathcal{P}$ and $\mathcal{R}$ as spaces of polynomials in $x_{i j}$. Similarly, we write $\partial_{i j}=\frac{\partial}{\partial x_{i j}}$ and consider the restrictions of the various differential operators,

$$
x=\left(x_{i j}\right), \partial=\left(\partial_{i j}\right), \gamma(x)=\operatorname{det}\left(x^{t} x\right), L=\operatorname{det}\left(\partial^{t} \partial\right), C=\gamma L .
$$

Let $H=G L_{k}^{+}(\mathbb{R})=\left\{g \in G L_{k}(\mathbb{R}) \mid \operatorname{det} g>0\right\}$ and define

$$
Y=X / H \text {. }
$$

The left action of the group $S O_{n}(\mathbb{R})$ on $Y$ is transitive and allows one to identify $Y$ with the compact symmetric space $S O_{n}(\mathbb{R}) /\left[S O_{k}(\mathbb{R}) \times S O_{l}(\mathbb{R})\right]$. The general structure of invariant differential operators on such a space is well understood, and we briefly recall the main ideas below. 


\section{Differential operators on symmetric spaces}

In this section we briefly recall some well-known results on compact symmetric spaces, referring the reader to [6] for details and proofs.

Let $K$ be a connected compact group with involution $\sigma$, let $K^{\sigma}$ denote the $\sigma$ fixed subgroup and let $\left(K^{\sigma}\right)_{0}$ denote its identity component. A subgroup $M$ of $K$ is said to be a symmetric subgroup if $\left(K^{\sigma}\right)_{0} \subset M \subset K^{\sigma}$, and in this case we say that $K / M$ is a symmetric space. In the case of interest to us $M=\left(K^{\sigma}\right)_{0}$ so we make this simplifying assumption in the subsequent discussion. Let $\mathfrak{k}, \mathfrak{m}$ denote the complexified Lie algebras of $K$ and $M$. The involution $\sigma$ on $K$ defines an involution, still denoted $\sigma$, on $\mathfrak{k}$ and we obtain the corresponding Cartan decomposition

$$
\mathfrak{k}=\mathfrak{m}+\mathfrak{s}
$$

into \pm 1 eigenspaces of $\sigma$. We fix a maximal abelian subspace $\mathfrak{a} \subset \mathfrak{s}$ and let $\mathfrak{t}$ denote the centralizer of $\mathfrak{a}$ in $\mathfrak{m}$. Then $\mathfrak{h}=\mathfrak{a}+\mathfrak{t}$ is a Cartan subalgebra of $\mathfrak{k}$. We let $\Sigma \subset \mathfrak{h}^{*}$ denote the root system of $\mathfrak{h}$ in $\mathfrak{k}$, with Weyl group $W=W(\Sigma)$, and fix a positive subsystem $\Sigma^{+}$. The irreducible representations of $K$ are classified by their highest weight $\lambda \in \Lambda$, where $\Lambda \subset \mathfrak{h}^{*}$ denotes the lattice cone of dominant integral elements.

The representations of $K$ with an $M$-fixed vector are those for which the highest weight $\mu$ belongs to the subcone $\Lambda_{0} \subset \Lambda$ consisting of even integral elements that vanish on $\mathfrak{t}$. Thus, if $\mathcal{H}$ denotes the space of $K$-finite functions on $K / M$, then we have a multiplicity free decomposition into irreducible $K$-modules

$$
\mathcal{H}=\bigoplus_{\mu \in \Lambda_{0}} \mathcal{H}^{\mu}
$$

It is convenient to work with the restricted root system $\Sigma_{0}=\left.\Sigma\right|_{\mathfrak{a}} \backslash\{0\} \subset \mathfrak{a}^{*}$, the restricted Weyl group $W_{0}$ and the positive subsystem $\Sigma_{0}^{+}=\left.\Sigma^{+}\right|_{\mathfrak{a}} \cap \Sigma_{0}$. We regard $\Lambda_{0}$ as a lattice contained in $\mathfrak{a}^{*}$ and we also define $\rho \in \mathfrak{a}^{*}$ by

$$
\rho=\frac{1}{2} \sum_{\alpha \in \Sigma_{0}^{+}} m_{\alpha} \alpha,
$$

where $m_{\alpha}$ denotes the multiplicity of the restricted root $\alpha$.

Let $\mathbf{D}(K / M)$ denote the algebra of $K$-invariant differential operators on $K / M$. Then one has an isomorphism, the Harish-Chandra isomorphism

$$
D_{0} \mapsto \Gamma_{D_{0}}: \mathbf{D}(K / M) \rightarrow \mathcal{I}(\mathfrak{a}),
$$

where $\mathcal{I}(\mathfrak{a}) \approx \mathcal{S}(\mathfrak{a})^{W_{0}}$ denotes the algebra of $W_{0}$-invariant elements in the symmetric algebra $\mathcal{S}(\mathfrak{a})$. We regard $S(\mathfrak{a})$ and $\mathcal{I}(\mathfrak{a})$ as spaces of the polynomial functions on $\mathfrak{a}^{*}$, and we write $\mathcal{I}_{d}(\mathfrak{a}) \subset \mathcal{I}(\mathfrak{a})$ for the space of invariant polynomials of degree $\leq d$. The key property of the Harish-Chandra homomorphism is as follows:

Proposition 3.1. If $D_{0} \in \mathbf{D}(K / M)$ has order $\leq d$, then $\Gamma_{D_{0}} \in \mathcal{I}_{d}(\mathfrak{a})$. Moreover, $D_{0}$ acts on $\mathcal{H}^{\mu}$ by the scalar $\Gamma_{D_{0}}(\mu+\rho)$.

\section{Differential operators on Grassmannians}

We specialize the results of the previous section to the case

$$
K=S O_{n}(\mathbb{R}), M=S O_{k}(\mathbb{R}) \times S O_{l}(\mathbb{R}), Y=K / M,
$$

assuming as before that $n=k+l$ and $k<l$. In this case the restricted root system is of type $B_{k}$ and we choose the usual positive subsystem

$$
\Sigma_{0}=\left\{ \pm e_{i} \pm e_{j}, \pm e_{j}\right\}, \Sigma_{0}^{+}=\left\{e_{i} \pm e_{j} \mid i<j\right\} \cup\left\{e_{i}\right\},
$$


where $e_{1}, \ldots, e_{k}$ are the unit vectors in $\mathfrak{a}^{*} \approx \mathbb{C}^{k}$. The root multiplicities are 1 for $e_{i} \pm e_{j}$, and $(l-k)$ for $e_{j}$. Thus we get

$$
\rho=\frac{1}{2} \sum_{i}[2(k-i)+(l-k)] e_{i}=\sum_{i}\left[\frac{n}{2}-i\right] e_{i},
$$

which we note agrees with formula (1.5). The lattice cone $\Lambda_{0}$ consists of integer $k$-tuples $\mu=\left(\mu_{1}, \ldots, \mu_{k}\right)$ satisfying

$$
\mu_{1} \geq \cdots \geq \mu_{k} \geq 0, \mu_{i} \equiv \mu_{j}(\bmod 2) \text { for all } i, j .
$$

The Weyl group $W_{0}$ acts on $\mathbb{C}^{k}$ by sign changes and permutations of the coordinates. Thus,

$$
W_{0} \approx S_{k} \ltimes(\mathbb{Z} / 2)^{k}
$$

and $\mathcal{I}=\mathcal{I}(\mathfrak{a})$ is the ring of polynomials in $k$ variables, invariant under permutations and sign changes. Now Proposition 3.1 specializes as follows:

Proposition 4.1. Let $D_{0} \in \mathbf{D}(Y)$ be an $S O_{n}(\mathbb{R})$-invariant differential operator of order $\leq d$, then $D_{0}$ acts on the space $\mathcal{H}^{\mu}$ by the scalar $\Gamma_{D_{0}}(\mu+\rho)$, where $\Gamma_{D_{0}}(\tau)$ is a polynomial of degree $\leq d$ in $k$ variables $\tau=\left(\tau_{1}, \ldots, \tau_{k}\right)$ which is invariant under permutations and sign changes.

\section{TWISTED DifFERENTIAL OPERATORS}

We continue with the notation $K=S O_{n}(\mathbb{R}), M=S O_{k}(\mathbb{R}) \times S O_{l}(\mathbb{R})$ and $Y=K / M$ as in the previous section, and also recall from Section 2 that

$$
X=M_{n, k}^{\prime}(\mathbb{R}), H=G L_{k}^{+}(\mathbb{R}), Y=X / H .
$$

Let $D$ be a $K \times H$ invariant differential operator of order $d$ on $X$. Then $D$ descends to a $K$-invariant differential operator $D_{0}$ on $Y=K / M$, whose Harish-Chandra image $\Gamma_{D_{0}}$ belongs to the space $\mathcal{I}_{d}=\{p \in \mathcal{I} \mid \operatorname{deg}(p) \leq d\}$. Now the function $\gamma=\operatorname{det}\left(x^{t} x\right)$ is strictly positive on $X$, hence for any real (or complex) number $s$, we can consider the twisted differential operator $\gamma^{-s} \circ D \circ \gamma^{s}$, which descends to a $K$-invariant differential operator $D_{s}$ on $Y$.

Theorem 5.1. If $D$ is a $K \times H$ invariant differential operator of order $\leq d$ on $X$, then the map $p_{D}: \mathbb{R} \rightarrow \mathcal{I}_{d}$ defined by $p_{D}(s)=\Gamma_{D_{s}}$ is a polynomial map in $s$ of degree $\leq d$. More precisely, there exist elements $c_{j}=c_{j, D} \in \mathcal{I}_{j}$ such that $p_{D}(s)=\sum_{j=0}^{d} c_{j} s^{n-j}$.

For the proof we need the following simple result:

Lemma 5.2. Let $V$ be a finite dimensional vector space and let $p: \mathbb{R} \rightarrow V$ be a smooth function such that for all $t, p(s+t)-p(s)$ is a polynomial of degree $\leq d-1$ in $s$. Then $p$ is a polynomial function of degree $\leq d$.

Proof. Taking the $d$ th order partial derivative of $p(s+t)-p(s)$ with respect to $s$, we deduce that for all $s, t$,

$$
p^{(d)}(s+t)-p^{(d)}(s)=0 .
$$

Substituting $s=0$, we conclude that $p^{(d)}(t)=p^{(d)}(0)$ is a constant for all $t$. It follows that $p^{(d+1)}(t)=0$, whence $p$ must be a polynomial of degree $\leq d$. 
Proof of Theorem [5.1. We proceed by induction on $d$. The result is clear for $d=0$, since an operator of order 0 is multiplication by a function, which by virtue of $K \times H$-invariance reduces to a constant, and hence belongs to $\mathcal{I}_{0}$. We now assume that $d>0$ and that the result holds for all invariant operators of order $\leq d-1$. Fix $t \in \mathbb{R}$ and define

$$
D^{\prime}=\gamma^{-t} \circ D \circ \gamma^{t}-D=\gamma^{-t}\left[D, \gamma^{t}\right] .
$$

Then $D^{\prime}$ is invariant by the first expression and is of order $\leq d-1$ by the last expression. Therefore, by induction $p_{D^{\prime}},(s)$ is a polynomial function of degree $\leq d-1$ taking values in $\mathcal{I}_{d-1}$. However, $D_{s}^{\prime}=D_{s+t}-D_{s}$ and so

$$
p_{D^{\prime}}(s)=\Gamma_{D_{s+t}}-\Gamma_{D_{s}}=p_{D}(s+t)-p_{D}(s) .
$$

Thus, we conclude that for all $t, p_{D}(s+t)-p_{D}(s)$ is a polynomial of degree $\leq d-1$ in $s$. From the definition, it is easy to see that $p_{D}(s)$ is a smooth function of $s$. It follows from the previous lemma that $p_{D}$ is a polynomial of degree $\leq d$ in $s$.

Thus, we have an expression $p_{D}(s)=\sum_{j=0}^{d} c_{j} s^{d-j}$ with $c_{j} \in \mathcal{I}_{d}$ and it remains to show that $c_{j} \in \mathcal{I}_{j}$ for all $j \leq d-1$. We proceed once again by induction on $d$. The case $d=0$ follows as in the previous paragraph. Let us consider the expansion of $h(s)=p_{D}(s+1)-p_{D}(s)$ :

$$
h(s)=\sum_{j=0}^{d-1} c_{j}^{\prime} s^{d-1-j}=\sum_{j=0}^{d} c_{j}(s+1)^{d-j}-\sum_{j=0}^{d} c_{j} s^{d-j} .
$$

It follows that $c_{j}^{\prime}=(d-j) c_{j}+$ a combination of $c_{k}$ for $k<j$. Inverting this we deduce that for $j \leq d-1$, we have

$$
c_{j}=\frac{1}{d-j} c_{j}^{\prime}+\text { a combination of } c_{k}^{\prime} \text { for } k<j .
$$

Now as explained in the previous paragraph, the polynomial $h$ is of the form $p_{D^{\prime \prime}}$ for the invariant operator $D^{\prime \prime}=\gamma^{-1} \circ D \circ \gamma-D$ of degree $d-1$. Therefore, by induction $c_{j}^{\prime} \in \mathcal{I}_{j}$ for all $j \leq d-1$, and by the above expression for $c_{j}$ we conclude $c_{j} \in \mathcal{I}_{j}$ as well.

Proposition 5.3. If $D$ is a $K \times H$ invariant differential operator of order $\leq d$ on $X$, then there is a polynomial $p_{D}(s, \tau)$ of total degree $\leq d$ such that $D_{s}$ acts on $\mathcal{H}^{\mu}$ by the scalar $p_{D}(s, \mu+\rho)$. Moreover, $p_{D}$ is invariant under permutations $\tau_{i} \leftrightarrow \tau_{j}$ and sign changes $\tau_{i} \rightarrow-\tau_{i}$.

Proof. This follows by combining Theorem 5.1 and Proposition 4.1

\section{Proof of Theorem 2.1}

We apply the result of the previous section to the invariant differential operator $C=C_{1,0}=\operatorname{det}\left(x^{t} x\right) \operatorname{det}\left(\partial^{t} \partial\right)$, which has degree $2 k$. By the previous proposition there exists a polynomial $p_{C}(s, \tau)$ of degree $\leq 2 k$ such that $C_{s}$ acts on $\mathcal{H}^{\mu}$ by the character $p_{C}(s, \mu+\rho)$.

Proposition 6.1. $C$ acts on $\mathcal{R}_{m}^{\mu}$ by the character $p_{C}\left(\frac{m}{2}, \mu+\rho\right)$.

Proof. Let $f \in \mathcal{R}_{m}^{\mu}$, then $\gamma^{-m / 2} f$ is $H$-invariant, hence $\gamma^{-m / 2} f \in \mathcal{H}^{\mu}$. Evidently, $\gamma^{-s} C=C_{s} \gamma^{-s}$, thus by the previous corollary we get

$$
\gamma^{-m / 2} C f=C_{m / 2}\left[\gamma^{-m / 2} f\right]=p_{C}\left(\frac{m}{2}, \mu+\rho\right)\left[\gamma^{-m / 2} f\right] .
$$


Multiplying both sides by $\gamma^{m / 2}$ we get the desired result.

In view of the above result, let us define

$$
q(s, \tau)=p_{C}\left(\frac{s-\rho_{1}}{2}, \tau\right) .
$$

Then by the previous proposition $C$ acts on $\mathcal{R}_{m}^{\mu}$ by the scalar $q\left(m+\rho_{1}, \mu+\rho\right)$, and Theorem 2.1 reduces to showing the following result.

Theorem 6.2. We have $q=q_{1,0}$.

We will prove this in two stages.

Proposition 6.3. There is a constant a such that $q=a q_{1,0}$.

Proof. Dividing $q$ by $s-\tau_{1}$ we obtain the expression

$$
q(s, \tau)=\left(s-\tau_{1}\right) h(s, \tau)+r(\tau) .
$$

Let $\mu \in \Lambda_{0}$ be arbitrary, then Lemma 2.2 implies that $C$ acts on $\mathcal{R}_{m}^{\mu}$ by 0 for $m=\mu_{1}$. Thus we get

$$
q\left(\mu_{1}+\rho_{1}, \mu+\rho\right)=0 .
$$

Substituting $s=\mu_{1}+\rho_{1}$ and $\tau=\mu+\rho$ in formula (6.2) we deduce that, for all $\mu \in \Lambda_{0}$,

$$
r(\mu+\rho)=0 .
$$

Now it is easy to see by induction on $k$ that the set $\Lambda_{0}+\rho$ is Zariski dense in $\mathbb{C}^{k}$. It follows that $r(\tau)$ is identically 0 , and thus $\left(s-t_{1}\right)$ divides $q$. Since $p_{C}$ and hence $q$ are invariant under permutations $\tau_{i} \leftrightarrow \tau_{j}$ and sign changes $\tau_{i} \rightarrow-\tau_{i}$, it follows that $\left(s \pm \tau_{i}\right)$ divides $q$ for all $i$. Thus $q_{1,0}=\prod_{i=1}^{k}\left(s^{2}-\tau_{i}^{2}\right)$ divides $q$, but since $\operatorname{deg} q \leq 2 k$ we must have $q(s, \tau)=a q_{1,0}$ for some constant $a$.

Now Theorem 2.1 reduces to proving $a=1$. For the proof we need a simple result on polynomials. In order to formulate and prove this result it is convenient to proceed in somewhat greater generality, thus we temporarily introduce the following notation:

$$
x=\left(x_{1}, \ldots, x_{n}\right), \partial_{i}=\partial / \partial x_{i}, \partial=\left(\partial_{1}, \ldots, \partial_{n}\right) .
$$

Let $g(x)$ be a polynomial, let $s$ be an additional variable and define $g^{s}(x)=[g(x)]^{s}$, considered as an analytic function on a suitable open set in $(x, s)$. Let $f(x)$ be a homogenous polynomial of degree $d$ and regard $f(\partial)$ as a constant coefficient differential operator of order $d$. We apply $f(\partial)$ to $g^{s}$ and then multiply the result by $g^{d-s}$ to obtain the expression

$$
g^{d-s}\left(f(\partial) g^{s}\right) .
$$

It is easy to see that this expression is a polynomial in $(x, s)$. We now have the following result where we use the symbol $\sim$ to denote equality modulo lower degree terms in $s$.

Lemma 6.4. In the setting of the previous paragraph, we have

$$
g^{d-s}\left(f(\partial) g^{s}\right) \sim s^{d} f(\nabla g),
$$

where $\nabla g=\left(\partial_{1} g, \ldots, \partial_{n} g\right)$ denotes the gradient of $g$. 
Proof. It suffices to prove the result when $f$ is a monomial. Thus we need to show that

$$
g^{d-s}\left[\partial_{i_{1}} \cdots \partial_{i_{d}} g^{s}\right] \sim s^{d}\left(\partial_{i_{1}} g\right) \cdots\left(\partial_{i_{d}} g\right)
$$

which follows easily by the chain rule and induction on $d$.

We now return to the general discussion.

Proposition 6.4. The constant a in Proposition 6.3 is 1.

Proof. Let 1 denote the constant function in $\mathcal{H}^{0}$. By the previous proposition we have

$$
C_{s} \mathbf{1}=q\left(2 s+\rho_{1}, \rho\right) \mathbf{1}=a q_{1,0}\left(2 s+\rho_{1}, \rho\right) \mathbf{1} \sim\left(a 4^{k}\right) s^{2 k} \mathbf{1} .
$$

On the other hand, recalling the definition of $C_{s}$, we get

$$
\gamma^{2 k-1}\left[C_{s} \mathbf{1}\right]=\gamma^{2 k-s}\left[\operatorname{det}\left(\partial^{t} \partial\right)\left(\gamma^{s}\right)\right] \sim s^{2 k} \operatorname{det}\left(y^{t} y\right),
$$

after applying the previous lemma and writing $y=\nabla \gamma$.

Now multiplying (6.3) by $\gamma^{2 k-1}$ and comparing with (6.4) we get

$$
s^{2 k} \operatorname{det}\left(y^{t} y\right) \sim s^{2 k}\left(a 4^{k}\right) \gamma^{2 k-1} .
$$

Let $I_{k}$ denote the $k \times k$ identity matrix, and let $J$ denote the $n \times k$ matrix $\left[\begin{array}{c}I_{k} \\ 0\end{array}\right]$, then we have $J^{t} J=I_{k}, \gamma(J)=1$. Moreover, an easy calculation shows that

$$
y(J)=\nabla \gamma(J)=2 J .
$$

Thus evaluating both sides of formula (6.5) at $x=J$ we get

$$
\operatorname{det}\left(4 I_{k}\right) s^{2 k} \sim\left(a 4^{k}\right) s^{2 k} .
$$

Since $\operatorname{det}\left(4 I_{k}\right)=4^{k}$, this proves $a=1$ as desired.

As noted above, this proves Theorem 6.2 and hence Theorem 2.1

\section{Appendix}

We apply the results of this paper to obtain identities in the algebra $\mathbf{D}(Y)$ of $K$-invariant differential operators on the symmetric space $Y=K / M$, where

$$
K=S O_{n}(\mathbb{R}), M=S O_{k}(\mathbb{R}) \times S O_{n-k}(\mathbb{R}) .
$$

As noted in Section 5 above we may also write $Y=X / H$, where

$$
X=M_{n, k}^{\prime}(\mathbb{R}), H=G L_{k}^{+}(\mathbb{R}) .
$$

Thus, if $D$ is a $K \times H$ invariant differential operator on $X$, then for any real (or complex) number $s$, the twisted differential operator $\gamma^{-s} \circ D \circ \gamma^{s}$ descends to an operator $D_{s}$ in $\mathbf{D}(Y)$. This applies in particular to the element $C_{d, d^{\prime}}=$ $\gamma^{d} \circ L^{d+d^{\prime}} \circ \gamma^{d^{\prime}}$ in (1.4), and we write

$$
C_{d, d^{\prime}, s}=\gamma^{-s} \circ C_{d, d^{\prime}} \circ \gamma^{s}=\gamma^{d-s} \circ L^{d+d^{\prime}} \circ \gamma^{d^{\prime}+s} .
$$

On the other hand, if $z$ belongs to the center $\mathcal{Z}(\mathfrak{k})$ of the enveloping algebra $\mathcal{U}(\mathfrak{k})$ of $\mathfrak{k}=\mathfrak{s o}(n)$, then $z$ gives rise to an operator $\mathbf{D}(Y)$ as well. A number of authors have given explicit construction of elements in $\mathcal{Z}(\mathfrak{k})$. In particular, we refer the reader to [11, Sections 6 and 7] for a certain element $D^{\dagger}(\lambda)=D_{F}(\lambda) \in \mathcal{Z}(\mathfrak{k})$ defined in terms of a noncommuting determinant, and shown in [11, p. 487] to coincide with another element constructed in terms of the Sklyanin determinant in [15]. 
Theorem 7.1. The following identity holds in $\mathbf{D}(Y)$,

$$
C_{d, d^{\prime}, s}=a_{d, d^{\prime}}(s) \prod_{j=0}^{d+d^{\prime}-1} D^{\dagger}\left(2\left(s+d^{\prime}-j\right)+n / 2-1\right)
$$

where $a_{d, d^{\prime}}(s)$ is a certain explicit constant.

Proof. Writing $C=C_{1,0}=\gamma \circ L$ and $C_{s}=\gamma^{1-s} \circ L \circ \gamma^{s}$ we have a factorization:

$$
C_{d, d^{\prime}, s}=C_{s-d+1} \cdots C_{s+d^{\prime}}=\prod_{j=0}^{d+d^{\prime}-1} C_{s+d^{\prime}-j} .
$$

Thus it suffices to show that for some explicit constant $a(s)$ one has

$$
C_{s}=a(s) D^{\dagger}(2 s+n / 2-1) .
$$

As before, let $\mathcal{H}$ denote the space of $K$-finite functions on $K / M$, and let $\mathcal{H}=$ $\bigoplus_{\mu \in \Lambda_{0}} \mathcal{H}^{\mu}$ denote its (multiplicity-free) decomposition into irreducible $K$-modules. It suffices to show that the two sides of (7.1) agree on each $\mathcal{H}^{\mu}$. Let $\rho_{i}=n / 2-i$ as before, then by (6.1), $C_{s}$ acts on $\mathcal{H}^{\mu}$ by the scalar

$$
p_{C}(s, \mu+\rho)=q\left(2 s+\rho_{1}, \mu+\rho\right)=\prod_{i=1}^{k}\left[\left(2 s+\rho_{1}\right)^{2}-\left(\mu_{i}+\rho_{i}\right)^{2}\right] .
$$

Now the action of $D^{\dagger}(\lambda)$ on $\mathcal{H}^{\mu}$ is given via the Harish-Chandra homomorphism,

$$
\bar{\gamma}: \mathcal{Z}(\mathfrak{k}) \rightarrow \mathcal{S}(\mathfrak{h})
$$

where $\mathfrak{h}$ is a maximal toral subalgebra in $\mathfrak{k}$, followed by evaluation at

$$
\left(\mu_{1}+\rho_{1}, \ldots, \mu_{k}+\rho_{k}, \rho_{k+1}, \ldots, \rho_{p}\right),
$$

where $p=\lfloor n / 2\rfloor$. By Theorem 7.2 of [11] we see that $D^{\dagger}(\lambda)$ acts on $\mathcal{H}^{\mu}$ by

$$
\begin{array}{cc}
\prod_{i=k+1}^{p}\left(\lambda^{2}-\rho_{i}^{2}\right) \prod_{i=1}^{k}\left[\lambda^{2}-\left(\mu_{i}+\rho_{i}\right)^{2}\right] & \text { if } n \text { is even, } \\
(\lambda-1 / 2) \prod_{i=k+1}^{p}\left(\lambda^{2}-\rho_{i}^{2}\right) \prod_{i=1}^{k}\left[\lambda^{2}-\left(\mu_{i}+\rho_{i}\right)^{2}\right] & \text { if } n \text { is odd. }
\end{array}
$$

Comparing this with (7.2) we obtain (7.1), which implies the result.

\section{REFERENCES}

[1] Michael Atiyah, Raoul Bott, and Vijay Kumar Patodi, On the heat equation and the index theorem, Invent. Math. 19 (1973), 279-330. MR0650828 (58 \#31287)

[2] Armand Borel, Essays in the history of Lie groups and algebraic groups, History of Mathematics, vol. 21, American Mathematical Society, Providence, RI, 2001. MR.1847105 (2002g:01010)

[3] Alfredo Capelli, Ueber die Zurückführung der Cayley'schen Operation $\Omega$ auf gewöhnliche Polar-Operationen, Math. Ann. 29 (1887), no. 3, 331-338, DOI 10.1007/BF01447728 (German). MR:1510419

[4] Sergio Caracciolo, Alan D. Sokal, and Andrea Sportiello, Noncommutative determinants, Cauchy-Binet formulae, and Capelli-type identities. I. Generalizations of the Capelli and Turnbull identities, Electron. J. Combin. 16 (2009), no. 1, Research Paper 103, 43. MR2529812(2010g:15003)

[5] William Fulton, Young tableaux, London Mathematical Society Student Texts, vol. 35, Cambridge University Press, Cambridge, 1997. With applications to representation theory and geometry. MR1464693 (99f:05119)

[6] Sigurdur Helgason, Groups and geometric analysis, Mathematical Surveys and Monographs, vol. 83, American Mathematical Society, Providence, RI, 2000. Integral geometry, invariant differential operators, and spherical functions; Corrected reprint of the 1984 original. MR.1790156(2001h:22001) 
[7] Roger Howe, Remarks on classical invariant theory, Trans. Amer. Math. Soc. 313 (1989), no. 2, 539-570, DOI 10.2307/2001418. MR986027 (90h:22015a)

[8] Roger Howe, Perspectives on invariant theory: Schur duality, multiplicity-free actions and beyond, The Schur lectures (1992) (Tel Aviv), Israel Math. Conf. Proc., vol. 8, Bar-Ilan Univ., Ramat Gan, 1995, pp. 1-182. MR1321638 (96e:13006)

[9] Roger Howe and Soo Teck Lee, Spherical harmonics on Grassmannians, Colloq. Math. 118 (2010), no. 1, 349-364, DOI 10.4064/cm118-1-19. MR2600534(2011c:22023)

[10] Roger Howe and Tōru Umeda, The Capelli identity, the double commutant theorem, and multiplicity-free actions, Math. Ann. 290 (1991), no. 3, 565-619, DOI 10.1007/BF01459261. MR 1116239 (92j:17004)

[11] Minoru Itoh, Capelli elements for the orthogonal Lie algebras, J. Lie Theory 10 (2000), no. 2, 463-489. MR 1774874 (2001k:17016)

[12] Minoru Itoh, Capelli identities for reductive dual pairs, Adv. Math. 194 (2005), no. 2, 345397, DOI 10.1016/j.aim.2004.06.010. MR2139918 (2006a:17010)

[13] Bertram Kostant and Siddhartha Sahi, The Capelli identity, tube domains, and the generalized Laplace transform, Adv. Math. 87 (1991), no. 1, 71-92, DOI 10.1016/00018708(91)90062-C. MR1102965 (92h:22033)

[14] Bertram Kostant and Siddhartha Sahi, Jordan algebras and Capelli identities, Invent. Math. 112 (1993), no. 3, 657-664, DOI 10.1007/BF01232451. MR1218328(94b:17054)

[15] Alexander Molev and Maxim Nazarov, Capelli identities for classical Lie algebras, Math. Ann. 313 (1999), no. 2, 315-357, DOI 10.1007/s002080050263. MR.1679788 (2000c:17013)

[16] Maxim Nazarov, Yangians and Capelli identities, Kirillov's seminar on representation theory, Amer. Math. Soc. Transl. Ser. 2, vol. 181, Amer. Math. Soc., Providence, RI, 1998, pp. 139163. MR1618751 (99g:17033)

[17] Andrei Okounkov, Quantum immanants and higher Capelli identities, Transform. Groups 1 (1996), no. 1-2, 99-126, DOI 10.1007/BF02587738. MR1390752 (97j:17010)

[18] Andrei Okounkov, Young basis, Wick formula, and higher Capelli identities, Internat. Math. Res. Notices 17 (1996), 817-839, DOI 10.1155/S1073792896000505. MR1420550(98b:17009)

[19] Siddhartha Sahi, The spectrum of certain invariant differential operators associated to a Hermitian symmetric space, Lie theory and geometry, Progr. Math., vol. 123, Birkhäuser Boston, Boston, MA, 1994, pp. 569-576. MR1327549 (96d:43013)

[20] Hermann Weyl, The classical groups, Princeton Landmarks in Mathematics, Princeton University Press, Princeton, NJ, 1997. Their invariants and representations; Fifteenth printing; Princeton Paperbacks. MR 1488158 (98k:01049)

Department of Mathematics, Rutgers University, New Brunswick, New Jersey

E-mail address: sahi@math.rutgers.edu

URL: http://www.math.rutgers.edu/ ${ }^{\text {sahi }}$ 